\title{
Twelve-pulse Rectifier Circuit Based on the PSIM Simulation Software
}

\author{
Xunzhe Wang
}

North China Electric Power University, Baoding,071000, China.

Keywords: phase-controlled rectifying circuit; PSIM simulation; twelve-pulse rectifying circuit; harmonic analysis

\begin{abstract}
This paper took the phase-controlled rectifying circuit as the research object to introduce the working principle of the three-phase rectifier circuit. Based on the PSIM software to build the circuit, this paper analyzed the working condition of the rectifier circuit under various common fire angles by taking the twelve-pulse rectifier circuit as the example and then compared the difference of the output voltage harmonic between the six-pulse rectifier circuit and twelve-pulse rectifier circuit via FFI. The PSIM simulation results showed that the twelve-pulse in series can reduce the pulsating component of the output voltage, decrease the current harmonics through the loads and then improve the power factor to some extent.
\end{abstract}

\section{Introduction}

The more pulse waves of the rectifier output voltage in a power cycle, the higher of the harmonic order in the output voltage with the corresponding smaller harmonic amplitude, then bring out better rectification characteristic. The twelve-pulse wave or even higher pulse wave polyphase rectifier circuit can be applied to reduce the effect of the rectifying device harmonic on the power grid. The phase-shift multiple connection, also called as multilevel, means to adopt various basic unit rectifier circuits with several stagger phrases. We can obtain the twelve-pulse rectifier circuit by two three-phase full controlled bridges in series. The PSIM simulation software was applied to obtain the simulation results of the twelve-pulse rectifier circuit and then compared it with the six-pulse rectifier circuit to gain the advantages of the twelve-pulse rectifier circuit.

\section{Simulation process}

The PSIM software is applied to build the following simulation graphics of the twelve-pulse rectifier circuit:

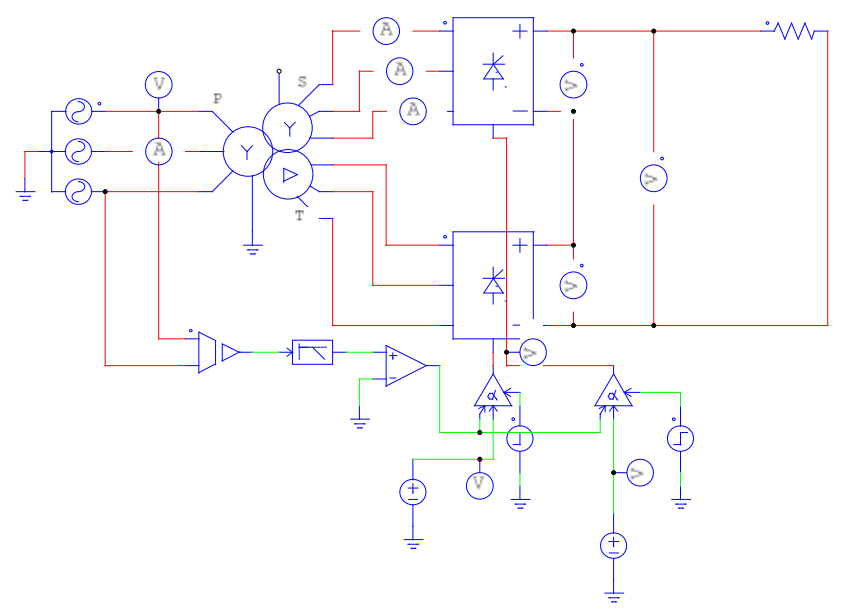

In order to compare the current waveform, we build the simulation graphics of the three-phase rectifier circuit: 


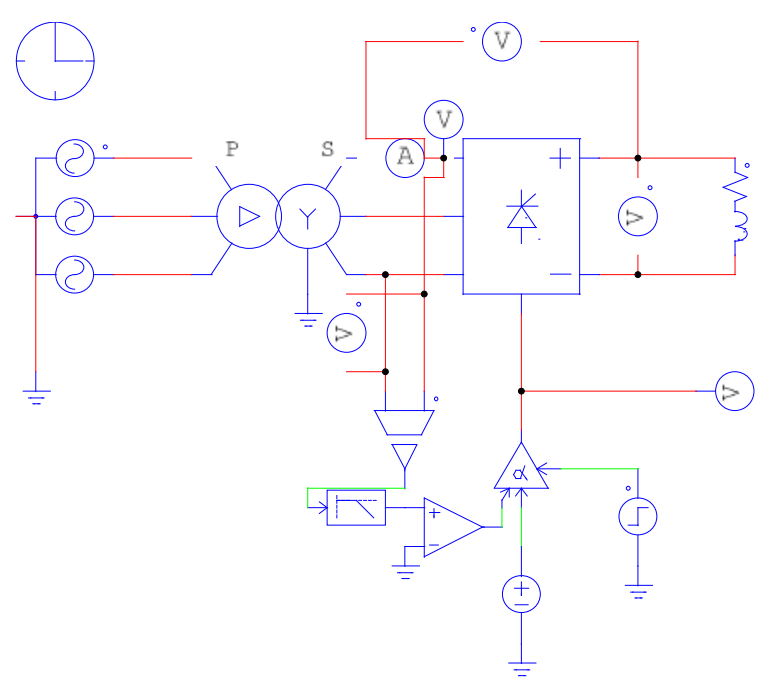

This paper takes the fire angles $\alpha=0^{\circ}, 30^{\circ}, 60^{\circ}$ to obtain the voltage waveform of star thyristor, triangle thyristor and load's voltage wave.

(1) $\alpha=0^{\circ}$
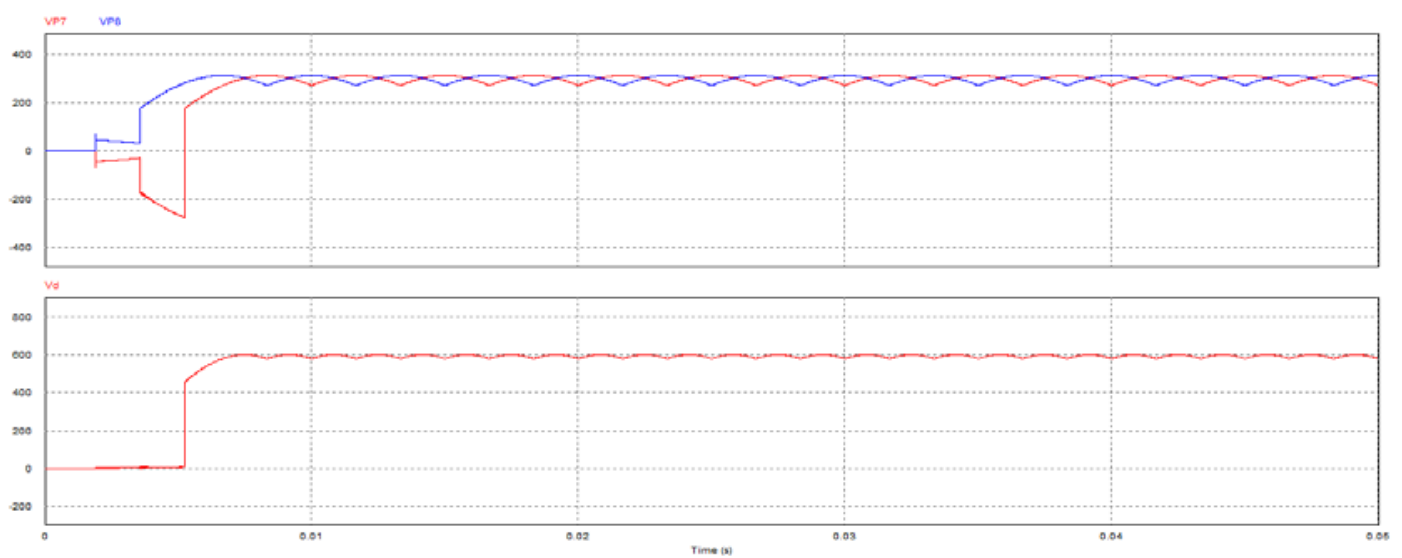

(2) $\alpha=30^{\circ}$
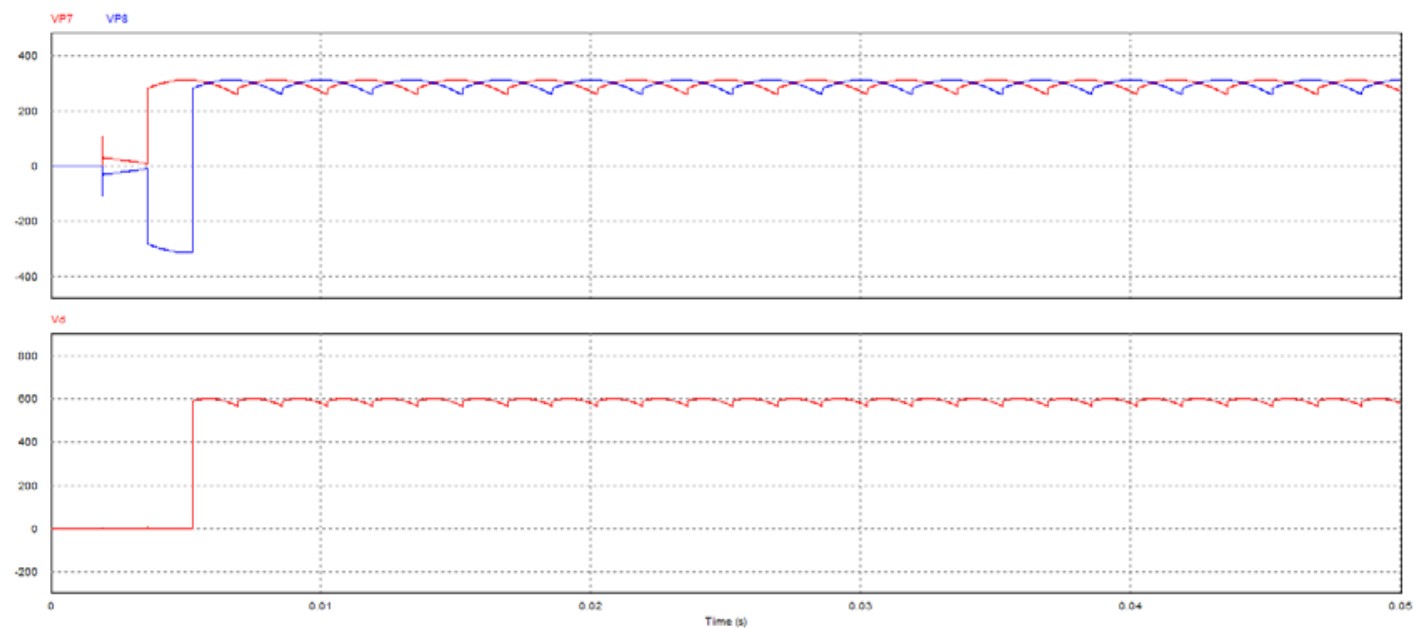
(3) $\alpha=60$
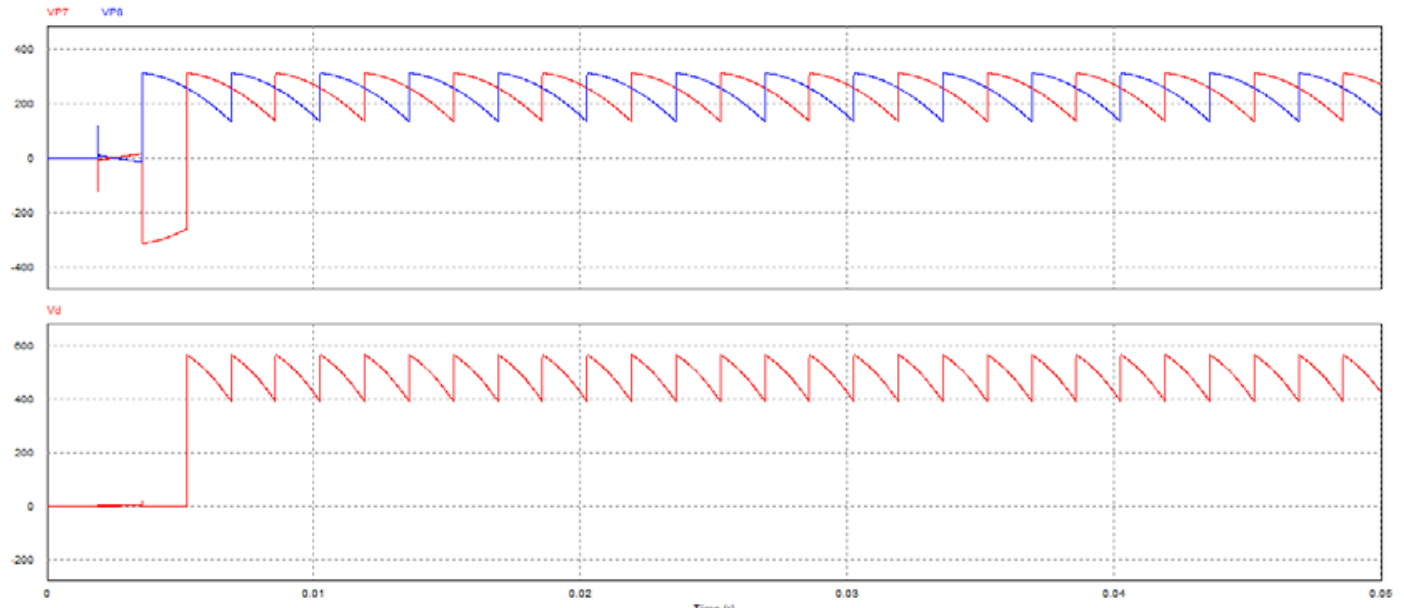

Observing the above graphics, it can be found that:

(1) The phase difference between $U_{Y}$ and $U_{d}$ was $30^{\circ}$; there appeared six waves in one cycle; hence its pulse-shake frequency was $300 \mathrm{~Hz}$; the load voltage $\mathrm{U}_{\mathrm{d}}$ has twelve waves in one cycle; hence its pulse-shake frequency was $600 \mathrm{~Hz}$.

(2) The average value of twelve-pulse output voltage in series was $U_{d}=U_{Y}+U$; namely, the output voltage of twelve-pulse wave was twice than the output voltage of six-pulse wave; hence the twelve-pulse is applicable for the output voltage.

(3) Compared with the output voltage waveform of six pulse wave, the output voltage waveform of the twelve pulse wave owned obviously smaller pulsating component.

\section{Analysis on the harmonic of six-pulse and twelve-pulse rectifier circuit}

When the fire angle $\alpha=60^{\circ}$, the output voltage of the twelve-pulse rectifier circuit and the six-pulse circuit were as follows:
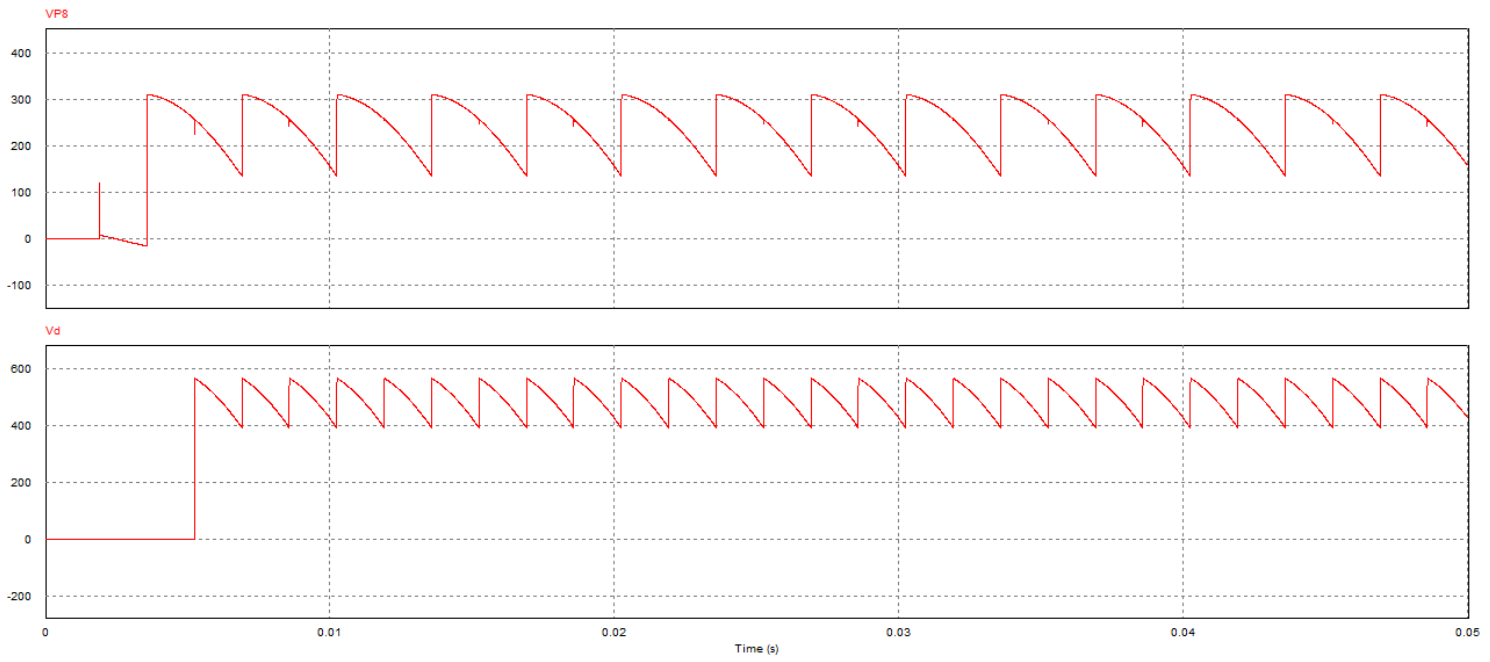
The Fourier transform was conducted on the waveforms to obtain the following graphics:
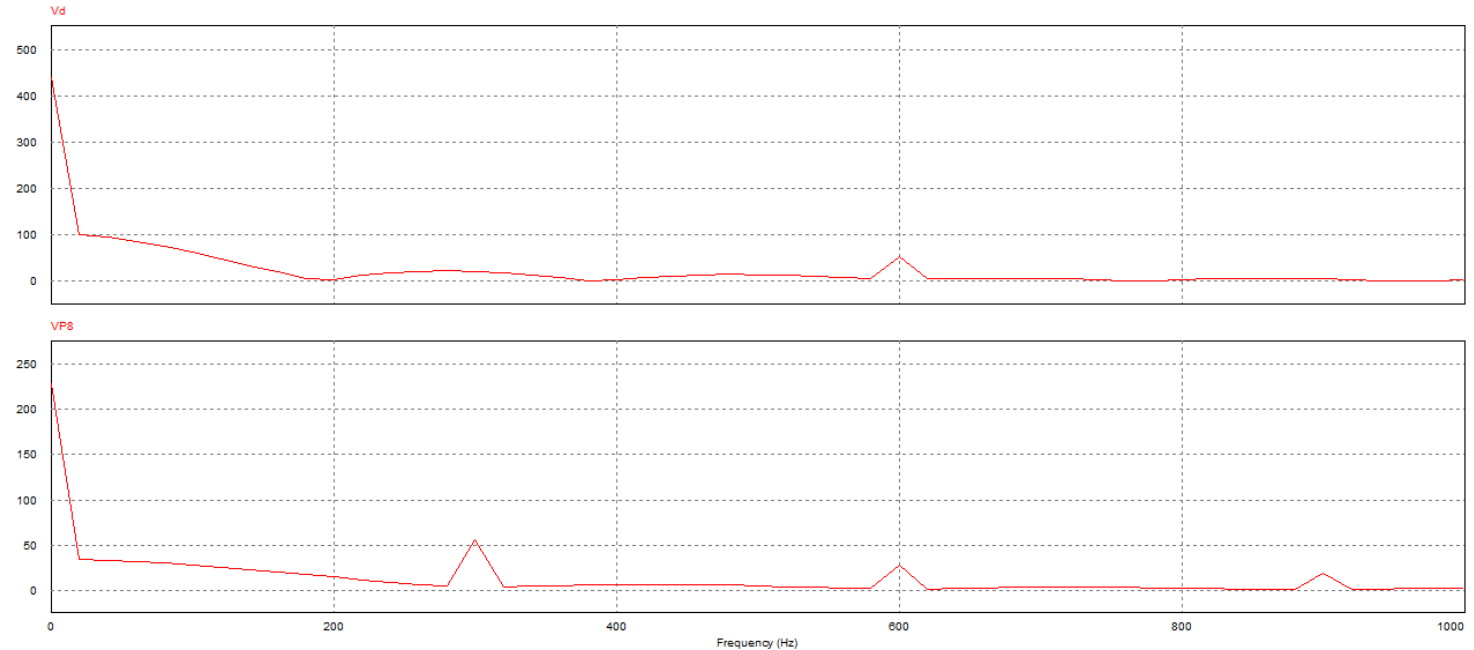

It can be observed that compared with the six-pulse rectifier circuit, the twelve-pulse rectifier circuit can reduce the harmonics of output voltage effectively by taking the Fourier harmonic analysis graphics within $1000 \mathrm{~Hz}$.

\section{Summary}

It can be seen from the above mentioned analysis that the twelve-pulse wave in series can reduce the pulsating component of the output voltage and decrease the current harmonics through the load and improve the power factor to some extent. When adopting the two full-controlled bridges in series, the output voltage was twice than one bridge output voltage. Therefore, this circuit can be widely applied in the high voltage, small current and the high-capacity load with relatively high power supply quality.

\section{References}

[1] Wang Zhaoan, Huang Ju. Power Electronic Technology, Beijing: China Machine Press, 2008

[2] Huang Ju, Qin Zuyin. Power Electronics Self-turn-off Device and Circuit, Beijing: China Machine Press, 1991

[3] Lin Weixun, Modern Power Electronics Technology, Beijing: China Machine Press, 2006

[4] Wang Weiping, Modern Power Electronics Technology and Application, Nanjing: Southeast University Press, 1999

[5] Yebin, Power Electronics Application Technology and Equipment, Beijing, China Railway Press, 1999

[6] Ma Jianguo, Meng Xianyuan, Technological Basis for the Electronic Design Automation, Beijing, Tsinghua University Press. 